\title{
DMRG on top of plane-wave Kohn-Sham orbitals: case study of defected boron nitride
}

\author{
Gergely Barcza, ${ }^{1,2, *}$ Viktor Ivády, ${ }^{1,3}$ Tibor Szilvási, ${ }^{4,5}$ Márton Vörös, ${ }^{6}$ Libor Veis, ${ }^{2}$ Ádám Gali, ${ }^{1,7}$ and Örs Legeza ${ }^{1}$ \\ ${ }^{1}$ Wigner Research Centre for Physics, Budapest, PO. Box 49, H-1525, Hungary \\ ${ }^{2}$ J. Heyrovsky Institute of Physical Chemistry, Czech Academy of Sciences, Prague, CZ-18223, Czechia \\ ${ }^{3}$ Department of Physics, Chemistry and Biology, \\ Linköping University, Linköping, SE-581 83, Sweden \\ ${ }^{4}$ Department of Chemical and Biological Engineering, \\ University of Wisconsin-Madison, Madison, WI-53706, USA \\ ${ }^{5}$ Department of Chemical and Biological Engineering, \\ The University of Alabama, Tuscaloosa, AL-35487, USA \\ ${ }^{6}$ Material Sciences Division, Argonne National Laboratory, Lemont, IL-60439, USA \\ ${ }^{7}$ Department of Atomic Physics, Budapest University of Technology and Economics, Budapest, H-1111, Hungary
}

(Dated: December 29, 2020)

\begin{abstract}
In this paper, we analyze the numerical aspects of the inherently multi-reference density matrix renormalization group (DMRG) calculations on top of the periodic Kohn-Sham density functional theory (DFT) using the complete active space (CAS) approach. The potential of the framework is illustrated by studying hexagonal boron nitride $(\mathrm{hBN})$ nano-flakes embedding a charged single boron vacancy point defect by revealing a vertical energy spectrum with prominent multi-reference character. We investigate the consistency of the DMRG energy spectrum from the perspective of sample size, basis size, and active space selection protocol. Results obtained from standard quantum chemical atom-centered basis calculations and plane-wave based counterparts show excellent agreement. Furthermore, we also discuss the spectrum of the periodic sheet which is in good agreement with extrapolated data of finite clusters. These results pave the way toward applying DMRG method in extended correlated solid state systems, such as point qubit in wide band gap semiconductors.
\end{abstract}

\section{INTRODUCTION}

By discovering a plethora of color centres in hexagonal boron nitride (hBN) with various point defect complexes in the last years ${ }^{1-7} \mathrm{hBN}$ is becoming a focus of interest. In particular, additionally to favourable optical properties, point defects in hBN with spin could possibly implement quantum bits controllable by optically detected magnetic resonance which signal has been recently reported at room temperature. ${ }^{8,9}$

The standard theoretical approach to study such defects is based on first-principles methods, ${ }^{10-22}$ in particular on the Kohn-Sham density functional theory (KSDFT), ${ }^{23}$ which established itself as one of the most prevalent numerical methods both in material science and theoretical chemistry offering balanced compromise between accuracy and computational demand. Despite its immense success attained in the field of weakly correlated systems, it is challenged to describe accurately quasidegenerate electronic structures exhibiting strong correlations. $^{24}$

To overcome the limitations of the standard DFT level of theory, various distinct computational frameworks have been proposed for molecular systems. ${ }^{25-34}$ The description of electron correlation effects in molecules are typically improved by configuration interaction based methods, coupled cluster theory, methods inspired by Monte-Carlo approach and variants of the complete active space self-consistent field approach, see e.g. ${ }^{35-43}$ More recently, the application of post-Hartree-Fock methods on periodic problems has witnessed significant progress as well. ${ }^{44-50}$ Besides these canonical approaches, in the last decade, density matrix renormalization group (DMRG) procedure also became an appealing alternative for describing the low-lying vertical excitation spectrum of strongly correlated molecules ${ }^{51}$ and periodic ab initio systems. ${ }^{52,53}$

DMRG was originally developed to describe onedimensional quantum models in solid state pyhsics with local interactions. ${ }^{54,55}$ The method was later generalized to treat long-range interactions found in momentum space representation of lattice models ${ }^{56-58}$ and in ab initio quantum chemistry. ${ }^{59-61}$ The success of these developments relies on the efficient factorization of interactions $^{56,59}$ and the optimization of the DMRG network topologies based on concepts of quantum information theory ${ }^{62-64}$ leading to tremendous reduction in computational costs. The underlying mathematical framework, however, is not restricted to models studied in condensed matter physics ${ }^{65,66}$ or applications to molecular clusters $^{51,64,67}$ but among many others it can be also used to study nuclear shell models, ${ }^{68-71}$, particles in confined potential $^{72-74}$ or problems in the relativistic domain. ${ }^{75-77}$ Therefore, mapping a physical problem to a proper model together with an optimal choice of basis could pave the road for DMRG applications in a broad range of disciplines which could surpass conventional methods.

In this work, we present the application of DMRG in a novel direction and use it as a post-DFT approach governed by the complete active space (CAS) protocol. ${ }^{78,79}$ The discussed approach focuses on the accurate description of the static correlations treating the rest of the electronic structure on effective one-electron level. The method, denoted as DFT-CAS-DMRG in the following, 
has been applied on top of atomic KS orbitals. ${ }^{80,81}$ Here, we apply the theoretical framework on top of both atomic and periodic KS orbitals to predict the main features of the low-lying many-body spectrum of molecular and periodic ab initio systems. In fact, quite recently, we have applied this post-DFT approach to the negatively charged boron vacancy $(\mathrm{VB})$ in single layer $\mathrm{hBN}^{82}$ confirming the experimental expectations on decay routes. ${ }^{8}$

As prequel of our former study, now we turn our focus to the technical aspects of this post-DFT approach adapted both to KS atomic and periodic orbitals. Particularly, we investigate the vertical many-body energy spectrum of a series of finite VB-hBN samples terminated with hydrogen atoms. As part of the study, to the best of our knowledge, we benchmark ab initio DMRG results on top of plane-wave based Kohn-Sham orbitals against the vertical spectrum computed in localized basis for the first time. Furthermore, tendencies observed on flakes are set against the results obtained for explicit periodic sheet.

The paper is structured as follows. In section II, we introduce shortly the DMRG method and also discuss the technical details of the Hamiltonian matrix construction and of the active space selection. In section III, we describe the main features of the studied molecular systems. In section IV, the numerical aspects of the simulations are summarized. In section $\mathrm{V}$, the results are discussed. Finally, conclusion is provided in section VI.

\section{METHODS}

\section{A. Quantum chemical Hamiltonian and molecular orbitals}

In this study, the molecular system of $N$ electrons with coordinate $\left\{\mathbf{r}_{i}\right\}$ is investigated in the Born-Oppenheimer approximation, i.e., in the presence of nuclei of atomic number $\left\{Z_{\mu}\right\}$ with fixed position $\left\{\mathbf{d}_{\mu}\right\}$. Considering the Coulomb interaction among charged particles, the corresponding time-independent Schrödinger equation,

$$
\hat{H} \Psi=\left[-\sum_{i}^{N}\left(\frac{1}{2} \nabla_{i}^{2}+\sum_{\mu} \frac{Z_{\mu}}{\left|\mathbf{r}_{i}-\mathbf{d}_{\mu}\right|}\right)+\sum_{i<j}^{N} \frac{1}{\left|\mathbf{r}_{i}-\mathbf{r}_{j}\right|}+\sum_{\mu<\nu} \frac{Z_{\mu} Z_{\nu}}{\left|\mathbf{d}_{\mu}-\mathbf{d}_{\nu}\right|}\right] \Psi\left(\mathbf{r}_{1}, \ldots, \mathbf{r}_{N}\right)=E \Psi\left(\mathbf{r}_{1}, \ldots, \mathbf{r}_{N}\right)
$$

is to be solved to obtain stationary electronic wave function $\Psi\left(\mathbf{r}_{1}, \ldots, \mathbf{r}_{N}\right)$ with energy $E$. Hamiltonian (1) measured in Hartree atomic units describes the kinetic energy of the electrons, their potential energy induced by the charged nuclei in addition to the electron-electron Coulomb interaction. Owing to the two-body interactions, the many-electron equation, Eq. (1), is not separable to a system of independent single-electron equations. An approximate mean field level solution can be provided by the variational Hartree-Fock method which treats Hartree and exchange interactions exactly but completely neglects correlation effects. Based on the Hartree-Fock solution, various methods has been developed to recover the correlation energy which methods may show limitations on accessible system size or accuracy. ${ }^{83}$ The density functional theory (DFT) can provide a viable compromise by mapping the original problem of interacting electrons to a gas of non-interacting particles in an effective potential which also includes the relevant effects of the electron-electron interactions in addition to the external potentials. In standard DFT procedure the energy is minimized with respect to the electron density obtained iteratively from the Kohn-Sham orbitals, $\left\{\psi_{j}(\mathbf{r})\right\}$. Whereas the Hartree potential is treated exactly in the DFT method, the accurate description of the many-orbital interactions, encoded in the exchangecorrelation energy functional, is the actual challenge of the approach. Various approximations for the functional had been suggested that were optimized in given con- densed matter systems but the method became only a standard in computational chemistry with the advent of hybrid functionals which include a portion of the exact exchange energy of the Hartree-Fock theory to give a more precise characterization of exchange effects.

The method has numerous advantages and became extremely popular for treating weakly correlated systems in the last decades. Notwithstanding the enormous success and broad applicability of the approach, one may have to go beyond the pure DFT approach to provide an accurate description of strongly-correlated electronic states. In the following, we summarize the simplest such possible procedure applicable on one-electron level theory based on the complete active space method.

\section{B. Matrix elements of the ab-initio Hamiltonian}

Considering large supercells, the electronic bands become flat, i.e., dispersionless in the reduced Brillouin zone. Furthermore, band states of $\Gamma$-point, which is the center of the Brillouin zone with zero momentum, are purely real-valued. Consequently, $\Gamma$-point states of large supercells can be regarded as molecular orbitals of solid state systems. Hence, similarly to molecular orbitals obtained by atomic codes, electronic states computed by periodic programs can also be employed to construct the corresponding ab initio Hamiltonian. The following 
discussion summarizes the computation of the Hamiltonian's matrix elements of spin restricted molecular orbitals and $\Gamma$-point only periodic states. In the following, these localized $\Gamma$-point periodic states are also referred to as orbitals. For the sake of simplicity, spin-restricted orbital set is considered but the concept can be readily generalized.

In terms of operators $\hat{a}_{i \sigma}^{\dagger}$ and $\hat{a}_{i \sigma}$, which creates and annihilates electron with $\sigma$ spin projection in orbital $i$, the second quantized representation of the ab initio Hamiltonian operator, Eq. (1), is parameterized as

$$
\hat{H}=\sum_{i j, \sigma} t_{i j} \hat{a}_{i \sigma}^{\dagger} \hat{a}_{j \sigma}+\frac{1}{2} \sum_{i j k l, \sigma \sigma^{\prime}} V_{i j k l} \hat{a}_{i \sigma}^{\dagger} \hat{a}_{j \sigma^{\prime}}^{\dagger} \hat{a}_{k \sigma^{\prime}} \hat{a}_{l \sigma}+E^{\mathrm{nuc}}
$$

with system specific one- and two-electron integrals, $t_{i j}$, and $V_{i j k l}$, respectively. Assuming all-electron description, the former integrals expand the kinetic contributions and the electron-nuclear attractions in the basis of electronic orbitals, $\left\{\psi_{j}(\mathbf{r})\right\}$,

$$
t_{i j}=-\int \psi_{i}^{*}(\mathbf{r})\left(\frac{1}{2} \nabla^{2}+\sum_{\mu} \frac{Z_{\mu}}{\left|\mathbf{r}-\mathbf{d}_{\mu}\right|}\right) \psi_{j}(\mathbf{r}) d \mathbf{r}
$$

with nuclei of atomic number $Z_{\mu}$ at fixed coordinate $\mathbf{d}_{\mu}$. Note that applying pseudopotentials, which is optional in atomic codes, but rather mandatory in planewave based calculations, the nuclei and the frozen core electrons are described by non-local potential functions. When states either in the band gap or close to it are considered, the correlation effects with core electron are negligible and the pseudopotential approximation is adequate. As a consequence of the approach, i.e., by reducing the number of electrons and making the core potential softer, plane-wave calculations become considerably less demanding.

The interelectronic repulsions yield two-electron integrals,

$$
V_{i j k l}=\int \frac{\psi_{i}^{*}(\mathbf{r}) \psi_{j}^{*}\left(\mathbf{r}^{\prime}\right) \psi_{k}\left(\mathbf{r}^{\prime}\right) \psi_{l}(\mathbf{r})}{\left|\mathbf{r}-\mathbf{r}^{\prime}\right|} d \mathbf{r} d \mathbf{r}^{\prime},
$$

whereas the nuclear-nuclear repulsion gives a constant energy shift,

$$
E^{\mathrm{nuc}}=\sum_{\mu<\nu} \frac{Z_{\mu} Z_{\nu}}{\left|\mathbf{d}_{\mu}-\mathbf{d}_{\nu}\right|}
$$

\section{Evaluation of the two-electron matrix elements}

The definition of the two-electron integrals (4) prescribes an expensive six dimensional real space integral. It is easy to show that the matrix elements can be equivalently evaluated by a three dimensional integral in reciprocal space $\mathbf{q}$,

$$
V_{i j k l}=\int \frac{\rho_{i l}(\mathbf{q}) \rho_{j k}(-\mathbf{q})}{|\mathbf{q}|^{2}} d \mathbf{q}
$$

using auxiliary density operators,

$$
\rho_{i l}(\mathbf{q})=\int \psi_{i}^{*}(\mathbf{r}) \psi_{l}(\mathbf{r}) \mathrm{e}^{\mathrm{i} \mathbf{q r}} d \mathbf{r} .
$$

We implemented the computation of Hamiltonian matrix elements within the plane-wave based Quantum Espresso suite $^{84,85}$ adopting the momentum space representation, Eq. (6), where the evaluation reduces to two wave function multiplications and Fourier transformation besides a threefold integration. Restricting ourselves to the usage of norm-conserving wave functions, we do not face the problem of augmentation charges observed in systems with ultrasoft pseudopotentials. ${ }^{86}$ In some situations, the divergence at $|\mathbf{q}| \rightarrow 0$ can be treated analytically. ${ }^{87} \mathrm{Nev}-$ ertheless, the application of large supercells ensures that the Fourier grid is fine enough to treat divergence safely by discarding $\mathbf{q}=0$ term in the numerical integration.

The number of two-electron matrix elements to be evaluated scales as quartic of the number of active orbitals thus their effective computation is the most critical part of constructing the Hamiltonian matrix. In order to optimize performance, balancing between memory size and numerical efforts, portions of the computationally expensive auxiliary operators, $\left\{\rho_{i l}(\mathbf{q})\right\}$, are cached during the two-electron matrix evaluation.

\section{DMRG}

In the following, the basic concept of the DMRG method is summarized, whereas interested reader finds thorough overviews of DMRG and related numerical approaches in the context quantum chemistry ${ }^{64,67}$

Many-body wave function $|\Psi\rangle$, i.e., an eigenstate of Eq.(2), expanded in the space of $L$ spatial-orbitals reads as

$$
|\Psi\rangle=\sum_{\{n\}} C^{(n)} \prod_{i=1}^{L}\left(\hat{a}_{i \uparrow}^{\dagger}\right)^{n_{i \uparrow}}\left(\hat{a}_{i \downarrow}^{\dagger}\right)^{n_{i \downarrow}}|0\rangle,
$$

with notation $n=\left(n_{1 \uparrow} n_{1 \downarrow} n_{2 \uparrow} n_{2 \downarrow} n_{3 \uparrow} n_{3 \downarrow} \ldots n_{L \uparrow} n_{L \downarrow}\right)$ where $n_{i \sigma} \in\{0,1\}$. The components of the state specific $C$ tensor increase exponentially with system size $L$ scaling as $2^{2 L}$. Hence, the exact solution of problem (2) on current classical machines is generally limited to systems represented with a dozen of orbitals.

It is shown ${ }^{88}$ that tensor $C$ is factorizable to a set of numerically more manageable matrices using the equivalent matrix product state (MPS) form of it, i.e.,

$$
C^{(n)}=\prod_{i=1}^{L} A_{i}^{\left(n_{i \uparrow} n_{i \downarrow}\right)} .
$$

The dimension of the $A_{i}$ matrix grows towards the center of the MPS chain as $\operatorname{dim}\left(A_{i}\right)=\left[4^{i-1}, 4^{i}\right]$ for $i \leq L / 2$ and $\operatorname{dim}\left(A_{i}\right)=\left[4^{L-i+1}, 4^{L-i}\right]$ otherwise (for even $L$ ). Consequently, recovering exact MPS representation of ten- 
sor $C$, the overall computational cost of the MPS matrices also grows exponentially just as the original tensor representation. To overcome this limit, the DMRG approach provides an approximate description of the $C$ tensor in terms of optimized matrices, $A_{i}^{\mathrm{DMRG}}$, truncated to a fixed manageable bond dimension, $M$, i.e., $\operatorname{dim}\left(A_{i}^{\mathrm{DMRG}}\right) \leq[M, M]$. Increasing $M$, the precision of the approximation is well controlled approaching variationally the exact solution. In the DMRG protocol the MPS matrices are locally optimized and truncated by minimizing the discarded entanglement between the left and right neighboring blocks of the MPS chain, obtained from the reduced density matrix of the block. The algorithm iterates through the MPS chain in sequential order back and forth until reaching convergence. For a detailed tutorial about the DMRG approach in MPS formalism, we refer to tutorial. ${ }^{66}$

Compared to wave functions obtained from typical quantum chemical approaches, the DMRG method parameterizes the eigenstates in terms of local variational objects instead of excitations of a reference configuration. Consequently, DMRG can precisely describe active spaces up to $40-80$ orbitals due to the implicitly polynomial scaling of computational demand for gapped noncritical systems. Most typically the method is applied to obtain the ground state properties but it can be used to describe not only the vertical but also the relaxed lowlying electronic excitations as well. ${ }^{89,90}$

In the following, we compute the vertical excitation spectrum on the relaxed ground state geometry. In the DMRG truncation procedure, the reduced density matrix of the blocks is formed of the equally weighted linear combination of all target states.

\section{Complete active space (CAS) method}

The investigated systems, consisting of dozens of atoms, is described by hundreds of Kohn-Sham orbitals which cannot be directly treated by the DMRG owing to the computational costs. Therefore, an optimal selection of orbitals with tractable size is needed which are responsible for the strong static correlations. The complete active space $(\mathrm{CAS})$ scheme $^{78,79}$ classifies the set of orbitals to three categories, i.e., the so called core and virtual orbitals are frozen to the mean field level and filled with two and zero electrons, respectively. The third class comprises of the so called active orbitals which are populated with the rest of electrons minimizing the energy.

Accordingly, the virtual orbitals does not play any role in the corresponding CAS Hamiltonian whereas the core electrons affect the electrons of the active space through the Coulomb interactions, i.e., the Hamiltonian of the active space reads

$$
\begin{aligned}
\hat{H}^{\mathrm{CAS}} & =E^{\mathrm{nuc}}+E^{\mathrm{core}}+\sum_{i j, \sigma} t_{i j}^{\mathrm{CAS}} \hat{a}_{i \sigma}^{\dagger} \hat{a}_{j \sigma} \\
& +\frac{1}{2} \sum_{i j k l, \sigma \sigma^{\prime}} V_{i j k l} \hat{a}_{i \sigma}^{\dagger} \hat{a}_{j \sigma^{\prime}}^{\dagger} \hat{a}_{k \sigma^{\prime}} \hat{a}_{l \sigma}
\end{aligned}
$$

with the previously defined $V_{i j k l}$ integrals restricted to the active orbital set. The one-electron integrals of the CAS space, $t_{i j}^{\text {CAS }}$, describes not only the kinetic energy of the active electrons and their attraction to nuclei but also their interaction with the core electrons. Describing the active electrons with the DMRG method, which treats exactly the electron exchange, the one-electron interactions are written as

$$
t_{i j}^{\mathrm{CAS}}=t_{i j}+\frac{1}{2} \sum_{c}\left(2 V_{i c c j}-V_{i c j c}\right)
$$

to treat the Coulombic effects of the frozen electrons on the active orbitals. Here, the summation runs only on the indices of the core orbitals. Finally, the additional energy contribution of the core electrons is summed up in term $E^{\text {core }}$, i.e,

$$
E^{\text {core }}=2 \sum_{c} t_{c c}+\sum_{c c^{\prime}}\left(2 V_{c c^{\prime} c^{\prime} c}-V_{c c^{\prime} c c^{\prime}}\right) \text {. }
$$

Including more and more orbitals in the active space, the corresponding CAS ground state energy gets lower and lower consistently, i.e., enlarging the active space class the energy of the CAS approaches variationally the ground state energy of the original problem.

In practice, the active space is restricted to the most important orbitals featuring strong correlation. Even though the method has limitations to provide correct description of dynamic correlations using relatively small active space, it captures static correlations with high accuracy providing valuable insight into the low-lying energy spectrum and the essential structure and symmetry properties of the corresponding electronic eigenstates.

Note also that, contrary to alternative post-DFT methods, ${ }^{91}$ the CAS Hamiltonian (11) to be investigated does not include the Kohn-Sham energies explicitly but only the Kohn-Sham orbitals by construction. Also, the absolute energies of the states computed from the CAS Hamiltonian are not trivially comparable with counterparts obtained on the DFT level of theory due to the different description of the exchange and correlation effects.

\section{E. Protocols for selecting the active space orbitals}

The studied systems are described by hundreds of occupied orbitals, which limits the applicability of sophisticated CAS selection schemes based on large-scale postself-consistent field calculations. ${ }^{92}$ Occupation pattern of natural orbitals ${ }^{93}$ evaluated on the level of secondorder Møller-Plesset perturbation theory ${ }^{94}$ or the complementing information of the occupied orbitals ${ }^{95}$ has 
been proven helpful guidance to construct minimal virtual space in periodic calculations as well.

Nevertheless, in the following, we use alternative scheme for constructing active subspace owing to two distinguished features of the investigated defect system. First, the performed DFT calculations provide a particularly accurate description of the ground state of the defects, hence its partial occupations on the level of perturbation theory are negligible and provide no insight into the structure of excited states. Second, in the studied defect systems, the low-lying many-body energy spectrum is expected to be conceptually described by the localized defect orbitals (see Fig. 1) and their interaction with the hosting environment, i.e., orbitals with large lobes around the central atoms can potentially interfere with the in-gap orbitals. Therefore, in the following, we apply two approaches to define the active space: i) we select canonical orbitals with prominent localization at the core of the defect, ii) we construct the active space based on energy window around the valence band maximum (VBM) level to verify the applicability of the CAS selection based on orbital localization around the defect.

It is to be noted that we do not localize the canonical orbital set in order to preserve their point group symmetry thus providing the possibility of studying the symmetry properties of the many-body states. In practice, such orbitals are taken into account whose degree of localization on the central part of the defect yield a critical value according to their orbital volumetric data or (in case of availability) to their projection on atomic basis. Comparing the many-body excited state spectrum yielded from the two CAS selection protocols, we find that the structure of the spectra is essentially identical up to some $0.001-0.2 \mathrm{eV}$ shifts in energy as discussed in section VB, i.e., the most important orbitals are the localized in-gap orbitals incorporated in both of the applied protocols. Nevertheless, obtaining slightly lower absolute energies with the applied variational computational procedure indicates that the orbital selection based on localization is preferable to the one based on KS energy. Correspondingly, results discussed in section $\mathrm{VC}$ are based on DMRG calculations performed on CAS which is selected according to orbital localization.

\section{INVESTIGATED SYSTEMS}

Defect-free hexagonal boron nitride monolayer has been investigated numerically on the level of ab initio dynamical mean-field theory using crystal atomic orbitals. The computed direct and indirect band gaps are of around $10 \mathrm{eV} .{ }^{96}$

Various defects embedded in hBN with potential in technology have been recently investigated, for the current progress see review. ${ }^{97}$ In this work, we study hBN nano-flakes and sheet hosting a negatively charged boron vacancy $(\mathrm{VB}-\mathrm{hBN})$. The defect system of $\mathrm{D}_{3 \mathrm{~h}}$ symmetry exhibits spin triplet ground state which can be un-
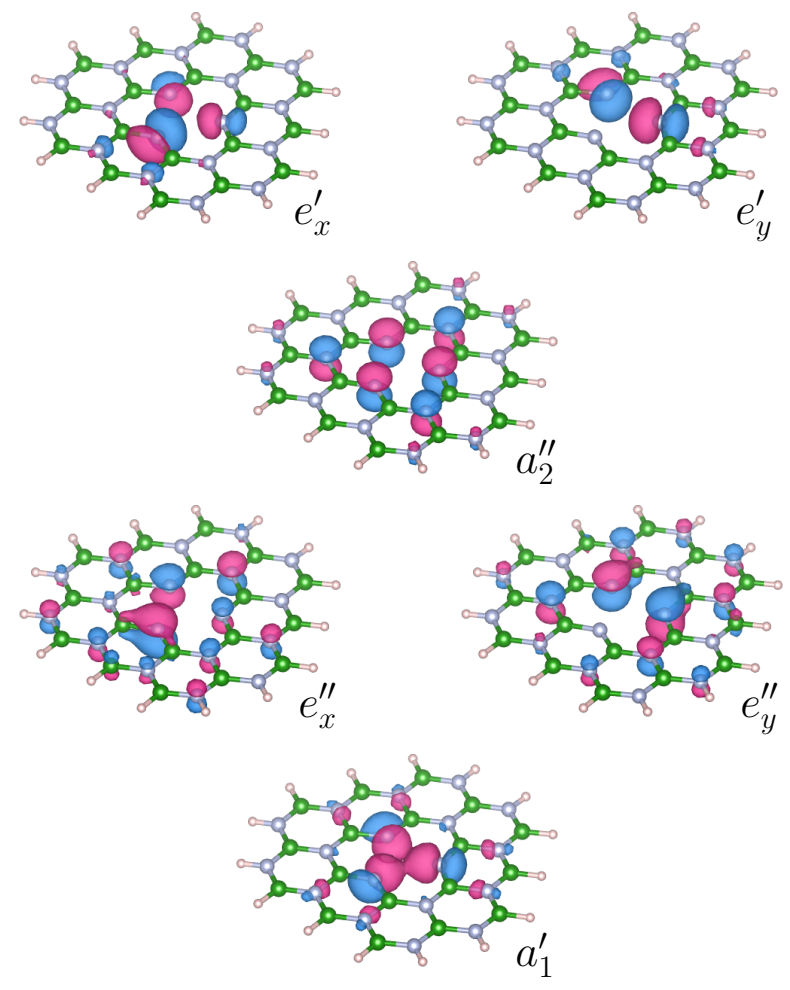

FIG. 1. The six defect molecular orbitals demonstrated for $\mathrm{B}_{18} \mathrm{~N}_{18} \mathrm{H}_{15}$ in cc-pVDZ basis. Defect orbitals characterized in Sect. III are localized dominantly on the core of the defect, i.e., on the three neighbor nitrogen atoms.

derstood as following. The in-plane dangling bonds and the out-of-plane $p_{z}$ orbitals of the three neighbor nitrogen atoms, located at the core of the defect, provide six single-particle defect states. The two non-degenerate $a$ and the two degenerate $e$ defect states plotted in Fig. 1 are occupied with ten electrons in the negatively charged system. The highest-lying $e$ dangling bond becomes half occupied yielding spin-triplet ground state. Analysing the single-electron spectrum of VB-hBN also reveals that these localized in-gap orbitals are close in energy. ${ }^{19}$

Therefore, the accurate description of the stronglycorrelated excited states necessitates the application of multi-reference methods. In our recent work, ${ }^{82}$ we provided a detailed description of magneto-optical properties of the system and also studied its many-body electronic spectrum applying the DFT-CAS-DMRG method. In this work, we turn our focus on the computational aspects of the DFT-CAS-DMRG approach illustrated on VB-hBN samples.

\section{COMPUTATIONAL DETAILS}

We study two-dimensional planar VB-hBN flakes of various sizes, i.e., investigating clusters of increasing size is necessary to capture long-range correlations and to minimize the finite-size effects of the terminating hydro- 


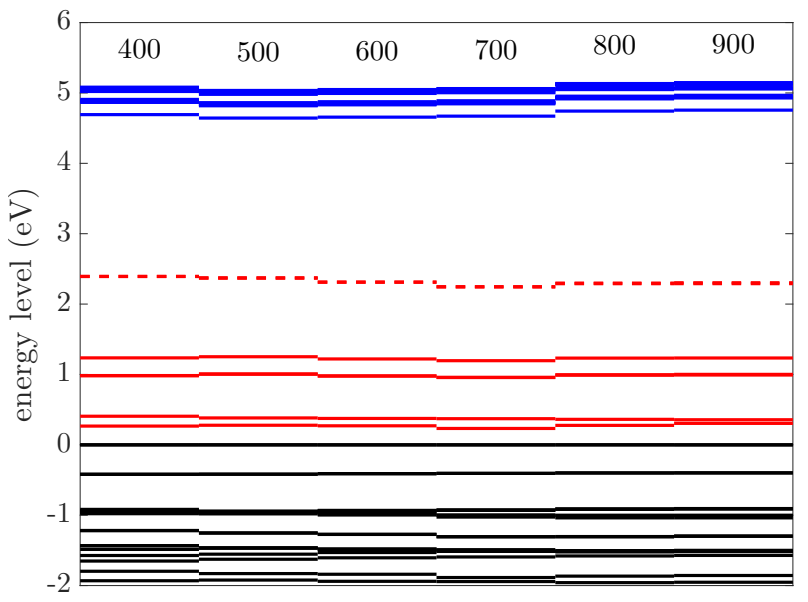

FIG. 2. Snippet of KS energy spectrum close to the VBM, for VB-hBN model with 18 boron atoms. DFT calculations on the DFT-PBE level of theory are performed using planewave basis with kinetic energy cutoff of the wave function at $\epsilon=400,500,600,700,800$ and $900 \mathrm{eV}$. Doubly and partially occupied in-gap orbitals are marked by solid and dashed red lines, whereas other valence and virtual orbitals are visualized by black and blue solid lines, respectively.

gen atoms. In particular, we study molecules $\mathrm{B}_{6} \mathrm{~N}_{6} \mathrm{H}_{9}$, $\mathrm{B}_{18} \mathrm{~N}_{18} \mathrm{H}_{15}, \mathrm{~B}_{36} \mathrm{~N}_{36} \mathrm{H}_{21}, \mathrm{~B}_{60} \mathrm{~N}_{60} \mathrm{H}_{27}, \mathrm{~B}_{90} \mathrm{~N}_{90} \mathrm{H}_{33}$. In addition, we investigate a periodic sheet of a 182 atom supercell which is large enough to minimize the interference between neighboring impurity sites and to reach convergence even restricting to $\Gamma$-point-only description.

The hosting molecular flakes embed the vacancy in the center of the model so that the relaxed lattice structures exhibit $\mathrm{D}_{3 \mathrm{~h}}$ symmetry. The relaxed molecular geometries of the charged defects are obtained on DFT-PBE level of theory ${ }^{98}$ with a homogeneous compensating background charge using VASP package. ${ }^{99}$ The finite flakes are embedded in a cubic supercell of $30 \AA$, correspondingly, $30 \AA$ vacuum size is applied in the perpendicular direction of the periodic sheet.

The electronic structure of the molecules is described in terms of spin restricted Kohn-Sham DFT orbitals using PBE functional. Self-consistent field (SCF) calculations are performed using both atomic basis based quantum chemical program suite $\mathrm{ORCA}^{100}$ and plane-wave based Quantum Espresso $(\mathrm{QE})^{85}$ with norm-conserving pseudo potentials. The actual capabilities of the periodic code are taken advantage of studying the single layer. Various atomic basis sets and plane-wave basis with increasing cutoff are tested as discussed in the Sect. V A.

The Hamiltonian matrix elements are computed by our in-house implementation for QE KS orbitals. Alternatively, matrix elements are also obtained from ORCA calculations through the interface to $\mathrm{MRCC}^{101,102}$ for isolated flakes. Active spaces of up to 40 orbitals are selected as discussed in Sect. IIE.

DMRG simulations are performed using the Budapest DMRG package. ${ }^{103}$ Due to the dozen of excited states to

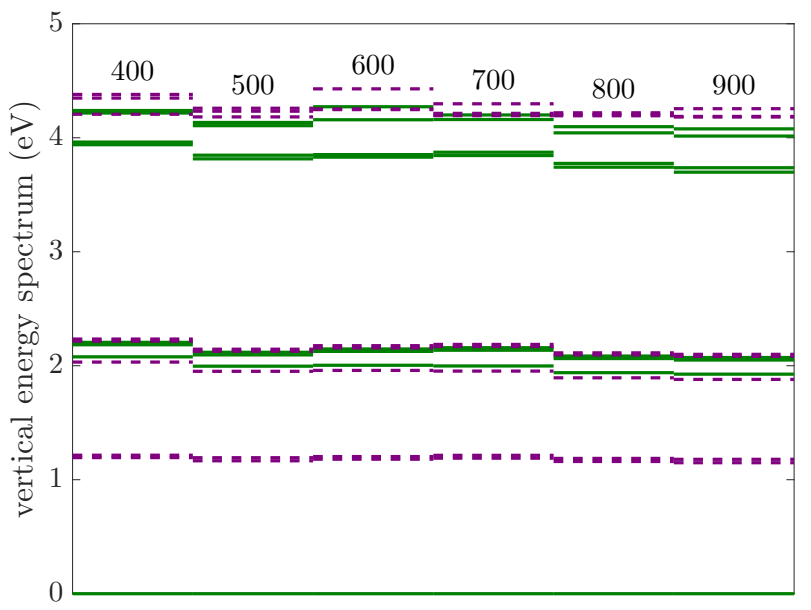

FIG. 3. Vertical energy spectrum relative to the ground state energy for model $\mathrm{B}_{18} \mathrm{~N}_{18} \mathrm{H}_{15}$. The active space is selected according to KS energy. Orbitals are expanded in PW basis with various energy cut-off $\epsilon$. For better visibility spin-triplet and spin-singlet energy levels are contrasted using solid green and dashed purple line, respectively.

be described, DMRG calculations are performed for target states with total spin 0 and 1 . In the DMRG truncation procedure, the density matrix is constructed of the equally weighted linear combination of all target states. As discussed in our tutorial, ${ }^{64}$ the convergence is sped up by initialization procedures inspired by quantum information theoretical considerations and the accuracy of the simulations is controlled by the dynamic block state selection approach. In the calculations, the quantum information loss is kept below threshold value $\chi=10^{-5}$. As the static correlation effects are dominantly attributed to the six defect orbitals, the optimal ordering is practically determined by the Kohn-Sham energies rendering the most strongly correlated orbitals to the center of the DMRG chain.

Note that the direct comparison with experimental data is not straightforward as the important structural relaxation effects are not incorporated in the current level of theory. Furthermore, it is also to be kept in mind that the neglected orbital relaxation effects as well as the accurate treatment of dynamical screening effects decrease the presented vertical excitation energies.

\section{RESULTS}

\section{A. Energy spectrum of $\mathrm{B}_{18} \mathrm{~N}_{18} \mathrm{H}_{15}$ obtained in localized and in plane-wave basis sets}

To demonstrate the robustness of the plane-wave based calculations, we investigate both the Kohn-Sham singleelectron and the resulted vertical many-body excitation spectrum of the model with 18 boron atoms in terms of the energy cutoff, which are plotted in Figs. 2 and 3, 


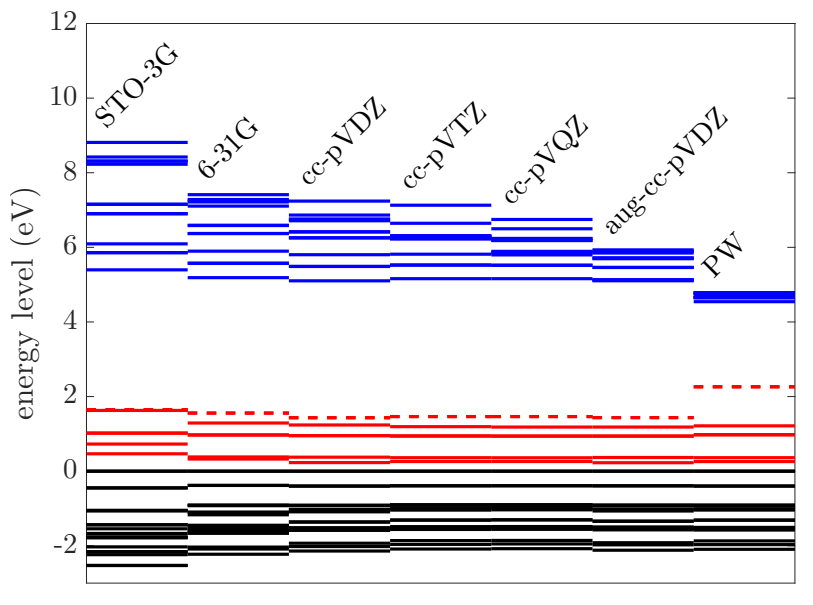

FIG. 4. Snippet of the Kohn-Sham one-electron energy spectrum of model $\mathrm{B}_{18} \mathrm{~N}_{18} \mathrm{H}_{15}$ on the DFT-PBE level of theory around the gap. Orbitals are expanded in STO-3G, 6-31G, cc-pVDZ, cc-pVTZ, cc-pVQZ, aug-cc-pVDZ atomic bases and in PW basis applying energy cutoff at $\epsilon=700 \mathrm{eV}$. Color key as in Fig. 2.

respectively. The kinetic energy cutoff of the wave function is varied in the $\epsilon=400-900 \mathrm{eV}$ range whereas the cutoff for charge density and potential is fixed as $4 \epsilon$ in agreement with the default QE parametrization. The details of the KS spectrum around the VBM are presented in Fig. 2 where the energy is measured relative to the lowest lying defect state. We find that the energy spectrum is rather independent of the applied energy cut-off which also illustrates the quick convergence of the DFT calculations. The observed small fluctuations are acknowledged to the fact that $\epsilon$ is increased in steps of 100 and not in steps of the lowest $\epsilon=400 \mathrm{eV}$ value. Correspondingly, due to the convergence of the $\mathrm{KS}$ orbitals observed already for surprisingly low cut-offs, the orbital-dependent DMRG results found in Fig. 3 vary marginally for $\epsilon>400 \mathrm{eV}$. Therefore, in the following, according to the observed quick convergence in cutoff energy, PW results for $\epsilon=700 \mathrm{eV}$ are used as reference data.

We also perform a series of DFT calculations for the model with 18 borons applying various complexity of atomic basis sets, ${ }^{78}$ i.e., STO-3G, 6-31G, cc-pVDZ, cc-pVTZ, cc-pVQZ, aug-cc-pVDZ. The corresponding Kohn-Sham one-electron spectra and, as reference, the result obtained in the plane-wave basis (PW) with energy cutoff of $\epsilon=700 \mathrm{eV}$ are presented in Fig. 4. Interestingly, whereas the details of the spectrum show some sensitivity to the applied basis, the energy gaps of the localized state are rather independent of the chosen atomic basis for 6-31G and more complex basis.

Comparing sophisticated atomic basis results to counterpart obtained in $\mathrm{PW}$, we find great agreement for the doubly occupied orbitals. The observed deviations are yielded from the different treatment of the core electrons and the effect of background charge in the PW calcula-

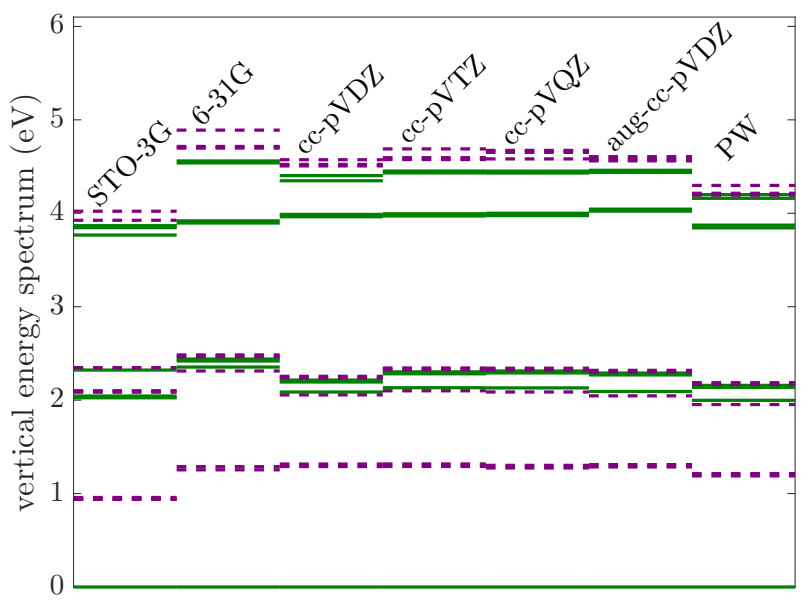

FIG. 5. Relative vertical energy spectrum of model $\mathrm{B}_{18} \mathrm{~N}_{18} \mathrm{H}_{15}$ obtained in active space of energy selected 21 orbitals which are expanded in STO-3G, 6-31G, cc-pVDZ, ccpVTZ, cc-pVQZ, aug-cc-pVDZ atomic bases and also in PW basis of $\epsilon=700 \mathrm{eV}$. Color code as in Fig. 3 .

tions. On the other hand, the KS energy of the partially occupied and the lowest lying virtual levels deviate significantly due to technical differences which understood as following.

The energy of half-filled defect $e^{\prime \prime}$ level displayed in Fig. 4 with dashed red line is discrepant corresponding to the distinct treatment of unpaired electrons applied by the atomic and by the plane-wave basis computations. In particular, the ORCA program describes the spin triplet ground state using restricted open-shell approach whereas the unpaired electrons are set to be smeared in spin averaged manner on the degenerate $e^{\prime \prime}$ level in the QE calculation. Therefore the observed energy difference corresponds to this spin flip energy. Nevertheless, we find that the corresponding ORCA and QE molecular orbitals are equivalent up to $95 \%$ comparing their volumetric data. Note that such disagreement on the level of the KS spectra does not affect the presented post-DFT results as the DFT-CAS-DMRG procedure does not rely on KS energies but exclusively on orbitals. The discrepancy of the virtual spectra is understood as the artifact of the PW calculations where the resulted lowest lying virtuals show a tendency to describe ionization, i.e., the corresponding orbitals are localized off the molecular sheet.

The many-electron DMRG energy spectrum resulted in the CAS constructed of the series of KS-DFT-SCF orbitals is plotted in Fig. 5. The active space for all the testing CAS calculations were selected according to the oneelectron energies correlating 32 electrons in 21 orbitals. The overall structure of the many-electron spectrum obtained in reasonably large basis sets, i.e., cc-pVDZ and beyond, is rather independent of the applied basis even for excitations of $4-5 \mathrm{eV}$. Using more and more sophisticated atomic basis, the absolute energy of each excitation lowers but with varying tendency. Nevertheless, approaching the completeness of the basis, i.e., using cor- 
relation consistent and $\mathrm{PW}$ basis with large cutoff $\epsilon$, the spectrum becomes consistent with slight variations yielding $\sim 90 \%$ agreement on the many-body excitation energies. It is also notable that the singlet-triplet gap, which involves predominantly spin excitation of the partially filled defect $e^{\prime}$ level, is predicted surprisingly well, i.e., with $10 \%$ accuracy, even on the minimal STO-3G level of theory. Based on these results, cc-pVDZ atomic basis is an optimal choice for reasonably accurate non-periodic DFT-CAS-DMRG calculations.

The observed discrepancies in the spectrum obtained for various orbital sets reflect not only the distinct character of the underlying incomplete basis sets but also the limitations of the CAS based description. In particular, as valence orbitals can be very close in energy with accidental ordering as observed in Fig. 4, the selection of a dozen of valence orbitals for the CAS can be biased which could be cured with the study of a much larger and optimally constructed active space.

\section{B. Sensitivity of active space selection}

Having a CAS based description in our hands, careful selection of active orbitals is crucial. In the following CAS selection is investigated from the perspective of active space size and selection protocols on the example of $\mathrm{B}_{36} \mathrm{~N}_{36} \mathrm{H}_{21}$ on cc-pVDZ level of theory.

First, the large gap, $\Delta \approx 5 \mathrm{eV}$, also observed in Fig. 4 and the partial occupation of the defect $e^{\prime \prime}$ level suggest that virtual orbitals does not play crucial role in the description of low-lying electronic states excited with energy of a few eV. Similar conclusion is drawn from the molecular orbitals obtained in PW SCF calculations, i.e., some of the lowest-lying virtuals are out of the plane of the sheet and expand in the vacuum, i.e., the system can be ionized with around energy $\Delta$.

Hence, we typically include only a couple of virtual orbitals in the active space and select active orbitals mostly from the valence band besides the six localized orbitals presented in Fig. 1. As an illustration, we show many-electron energy spectrum obtained for KS-energyselected active space of various sizes in Fig. 6. Increasing CAS size as more and more correlation effect is possible to be retrieved, consequently every many-electron energy decreases but with a particular rate depending on the structure of the corresponding many-body state. We find that the main features of the spectrum are already captured using the six orbital CAS, nevertheless the energy of some many-body states lower with more than $1 \mathrm{eV}$ including more dynamic correlations by increasing CAS size. The results also show that a CAS of around 30 orbitals is already large enough to describe the low-lying application-relevant excitations. It is notable that besides the ground state, i.e., $\approx 1 e^{\prime 2}$ in hole representation, there are some many-body excited states which are predominantly characterized by defect-orbital excitations whose energy decreases less dramatically with

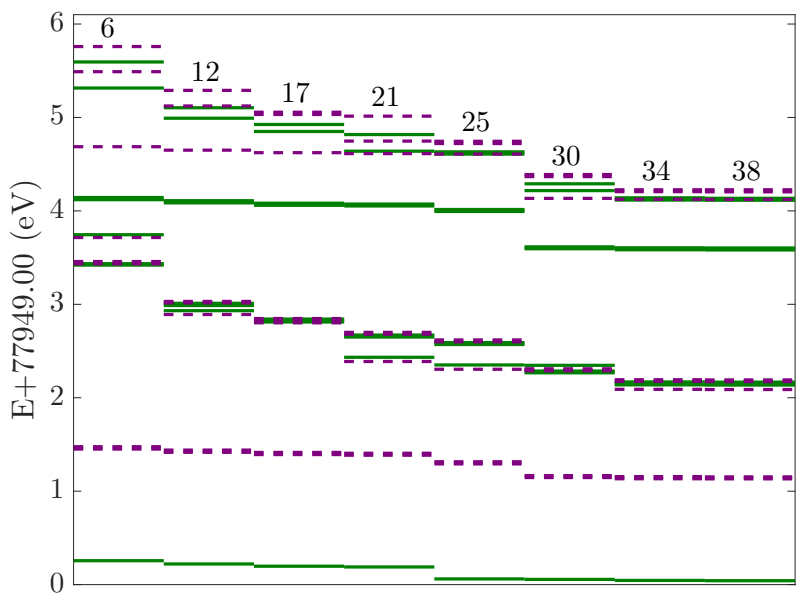

FIG. 6. Many-electron energy spectrum of model $\mathrm{B}_{36} \mathrm{~N}_{36} \mathrm{H}_{21}$ obtained for CAS with $6,12,17,21,25,30,34$ and 38 orbitals selected according to KS energy on DFT-cc-pVDZ-PBE level of theory. Energies are shifted for better visibility. Color code as in Fig. 3.

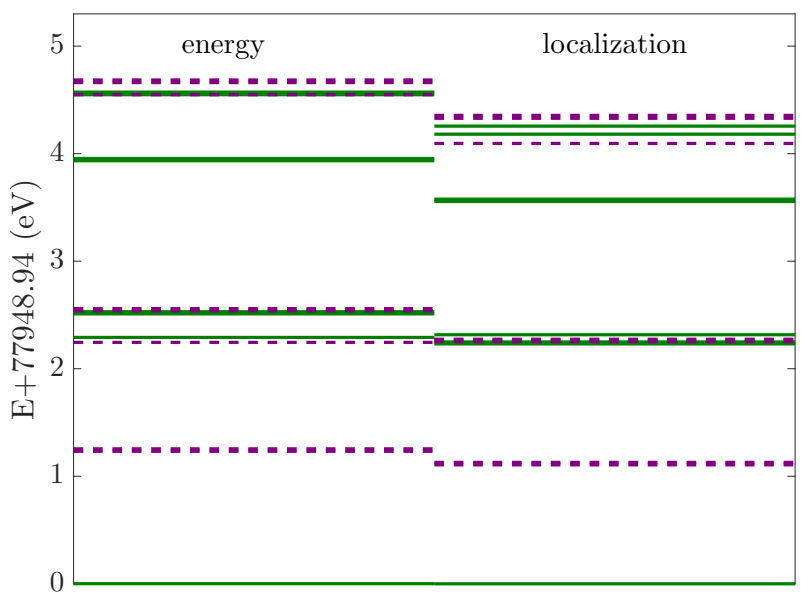

FIG. 7. Many-electron energy spectrum of model $\mathrm{B}_{36} \mathrm{~N}_{36} \mathrm{H}_{21}$ on DFT-cc-pVDZ-PBE level of theory obtained for CAS of 25 orbitals based on KS energy and localization as discussed in Sect. II E. Energies are shifted for better visibility. Color code as in Fig. 3.

increasing active space. In particular, the lowest-lying spin-singlet spatial-doublet state is dominantly characterized by spin excitations within the degenerate defect $e^{\prime}$ level, i.e., $\approx 0.7 e^{\prime 2}+0.3 e^{\prime} a_{1}^{\prime}$ in hole representation as we have shown. ${ }^{82}$ The multiple quasi degenerate states found around $2 \mathrm{eV}$ are also of multi-reference character. In particular, spatial doublets are detected with both spin singlet and triplet symmetry which are dominated by multiple configurations summarized as $\approx 0.45 e^{\prime} a_{2}^{\prime \prime}+0.45 e^{\prime} e^{\prime \prime}$ in hole representation. Considering higher-energy excitations, e.g., the spin-triplet doublet state with singlereference character found around $4 \mathrm{eV}$ for smaller active space sizes, i.e. $<30$, describes excitation from the localized $a_{1}^{\prime}$. Nevertheless, extending the active space 


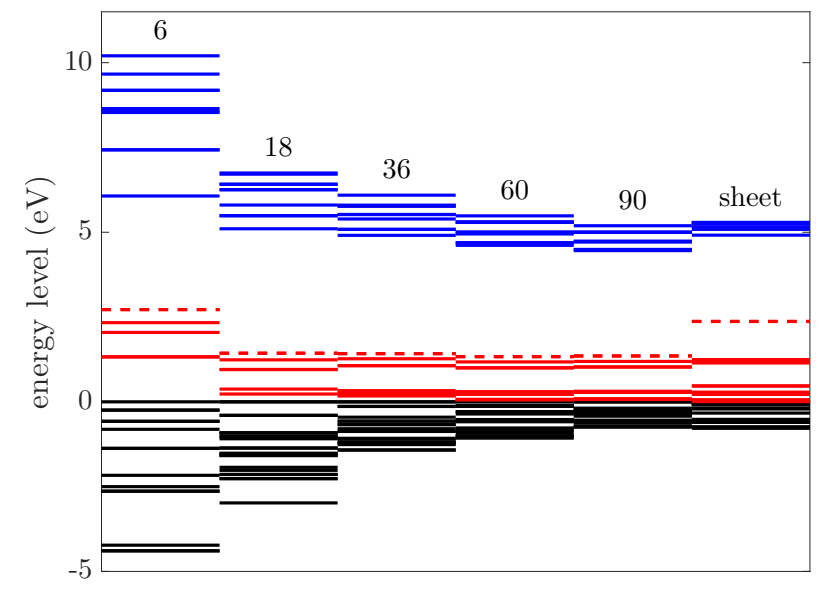

FIG. 8. Snippet of the KS energy spectrum of orbitals around the gap on the DFT-PBE level of theory for models with 6 , $18,36,60,90$ boron atoms and for the periodic sheet with 80 boron atoms computed using cc-pVDZ atomic and planewave basis with cut-off at $700 \mathrm{eV}$, respectively. Color key as in Fig. 2.

further with lower-energy orbitals, which exhibit significant localization around the defect, the state of character $\approx 0.95 e^{\prime} a_{1}^{\prime}$ shifts by $0.5 \mathrm{eV}$ for active spaces of $\geq 30$ orbitals.

This observation also underlines the detailed analysis of the eigenstates, which indicates that valence orbitals showing larger overlap with the defect orbitals become more correlated in the post-DFT calculations than orbitals far from the center confirming the chemical intuition of CAS selection based on localization. In Fig. 7, we demonstrate the results obtained for 25 active orbitals selected by the two CAS construction schemes, i.e., selecting orbitals based on their KS energy and on their localization around the defect. In the first case the occupied orbitals are kept in the active space based solely on their proximity to the Fermi surface. In the latter case the localization filters further the orbitals, i.e., only those orbitals are considered whose atomic basis projection on the first neighbor three nitrogen atoms reaches at least 0.005-0.01. Comparing DMRG spectrum obtained in the two differently constructed active spaces of 25 orbitals, corroborates the expectations, i.e. all the many-electron states get lower in energy with $0.0001-0.2 \mathrm{eV}$ using localization based CAS. In fact, the latter calculation recovers essentially the spectrum obtained for the larger CAS of 30 orbitals selected according to KS energy (see Fig. 6). In larger systems, where the corresponding orbitals are more distributed in space, the CAS selection scheme has even more drastic effect.

\section{Large model and single sheet results}

The KS-PBE energy levels close to the gap are depicted in Fig. 8 for various model sizes and for periodic

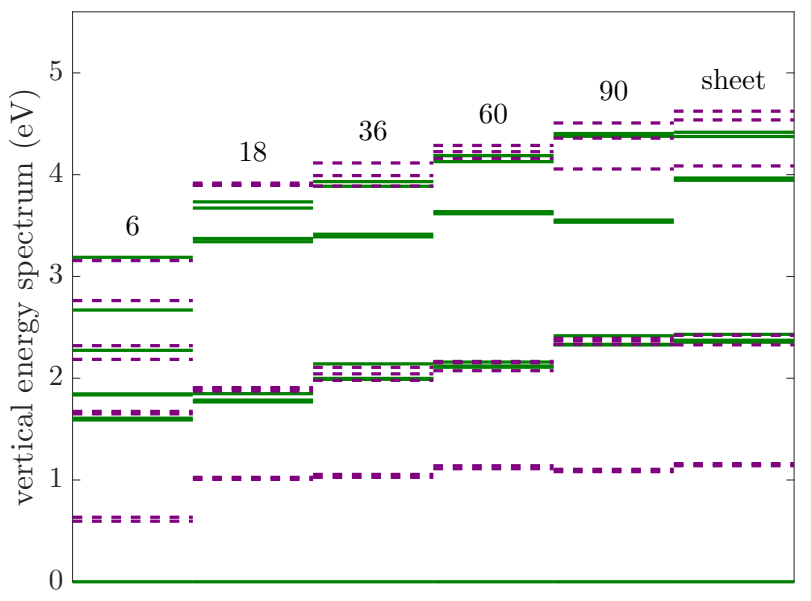

FIG. 9. Relative vertical energy spectrum of models with $6,18,36,60,90$ boron atoms and of single layer with 80 borons obtained in localization-selected CAS of 40 orbitals. The models and the sheet are described in cc-pVDZ and PW basis, respectively. Color code as in Fig. 3.

sheet with 80 boron atoms. Having observed good convergence on the relatively cheap cc-pVDZ basis set for $\mathrm{B}_{18} \mathrm{~N}_{18} \mathrm{H}_{15}$ in Sect. V A, all molecules are treated on the cc-pVDZ level of theory whereas the periodic sample is computed using PW basis with cut-off at $\epsilon=700 \mathrm{eV}$. We find that the structure of the in-gap spectrum is similar for models with 18, 36, 60 and 90 boron atoms, however, the energy gaps are enlarged in smaller models due to the quantum confinement. It is also clearly observed that the confinement affects strongly the typical extended molecular orbitals but hardly the six localized defect states which are coded essentially with color red in Fig. 8. Furthermore, we also find that the doubly occupied energy spectrum of the larger molecules is in good agreement with result obtained on the periodic sheet (also noting differences of partial-filled and virtual energies owing to technical reasons discussed in Sect. V A.

The yielded vertical many-electron DMRG spectrum obtained for CAS with 40 localized orbitals in the active space is presented in Fig. 9 for the distinct systems. The ground state has single reference nature in each cases, i.e. $>0.95 \%$ weight for the leading configuration with halffilled $e$ orbitals is observed, confirming the applicability of DFT as ground-state calculation on VB-hBN systems. Disregarding the smallest model with 6 borons, we find that the overall structure of the vertical many-body spectrum is largely independent of system size. Nevertheless, the actual excitation energies generally increase with system size yielding convergence for model with $60-90$ boron atoms for lower-lying excitations. Interestingly, the minimal excitation energy, i.e., singlet-triplet energy gap, is less dependent on the model size. Its stability is the consequence of the corresponding many-body state, which is dominated by the spin flip on the half-filled twofold degenerate $e$ orbitals.

Furthermore, comparing spectra obtained on the peri- 
odic sheet with 80 boron atoms and larger flake models in Fig. 9, rather good agreement is observed. In particular, for excitations below $3 \mathrm{eV}$, which are actually the most relevant for possible quantum bit applications, ${ }^{8}$ the agreement is quantitative. Therefore, the results indicate that clusters with $60-90$ borons can already reproduce the low-energy features of large periodic sheets, whereas the smaller flakes, especially $\mathrm{B}_{6} \mathrm{~N}_{6} \mathrm{H}_{9}$, have limitations to capture the physics of the single layer due to the nonnegligible perturbative effect of the terminating hydrogen atoms.

The effect of the active space selection on the electronic spectrum, i.e., the relevance of dynamic correlation is investigated as well. First, in addition to the presented 40 orbital calculations, we performed further DMRG computations on 25 orbitals selected according to localization concluding very similar many-body energy spectrum. In fact, for models with 6-18 borons the variation is insignificant for all many-body states whereas for larger systems the energies shift with up to $5 \%$ for the higher excitations. Investigating the spectrum resulted of $10-15$ relevant orbitals, more significant deviations of $10-15 \%$ are observed in the excitation regime above $3 \mathrm{eV}$ for larger systems. These results suggest that active space of 30-40 orbitals might be already sufficient to provide reasonable description for the low-energy excitations. Nevertheless, the accurate description of the missed portion of dynamic correlations could potentially lower the gaps of larger systems to some extent.

\section{CONCLUSION}

We report the first detailed implementation and discussion of DFT-CAS-DMRG method on plane-wave basis. As application of the approach, we investigate the electronic structure of VB-hBN defect systems of current interest, i.e., the sheet with a single defect and its molecular models. Applying our novel computational scheme to construct the CAS Hamiltonian of QE-DFT orbitals, we find great agreement on the many-electron spectrum with results obtained using standard atomic quantum chemical program suite.

We show that flakes with around 60-90 boron atoms are large enough to reflect faithfully the low-lying energy spectrum of the sheet with 80 borons. In agreement with expectations, we confirm that static correlation effects can be attributed mainly to the six localized defect orbitals and using CAS of 30-40 orbitals can provide a rea- sonable description of the main electronic features of the system. Further dynamic correlation effects retrieved by the application of perturbative methods ${ }^{104}$ would slightly modify the spectrum. Nevertheless, the presented conclusion on convergence issues are in agreement with recent analysis conducted on substitutional nitrogen defect neighbored with nitrogen vacancy in h-BN sheet using various computational schemes. ${ }^{105}$

The simulation of the periodic sheet also serves as validation and benchmark of the PW based approach whose actual potential advantage is to be realized in bulk calculations. Given the underlying theoretical background of the presented DFT-CAS-DMRG method, we hypothesize that it might be well-suited for predicting the main features of the low-lying many-body spectrum of wideband-gap semi-conducting defect materials with localized ingap orbitals which class of materials show emerging potentials in various quantum technology applications.

\section{ACKNOWLEDGEMENTS}

GB acknowledges the support of the NKFIH PD-17125261, NKFIH FK-20-135496 projects and of the Bolyai Research Scholarship of the Hungarian Academy of Sciences. VI is supported by the MTA Premium Postdoctoral Research Program and by the Knut and Alice Wallenberg Foundation through WBSQD2 project (Grant No. 2018.0071). LV acknowledges the support from Czech Science Foundation (grant no. 18-18940Y). ÖL and AG acknowledge support of the NKFIH through the National Quantum Technology Program (Grant No. 2017-1.2.1-NKP-2017-00001). ÖL acknowledges grant NKFIH K120569 and the support of the Alexander von Humboldt Foundation. AG acknowledges the Hungarian NKFIH grants No. KKP129866 of the National Excellence Program of Quantum-coherent materials project. The development of the DMRG libraries was supported by the Center for Scalable and Predictive methods for Excitation and Correlated phenomena (SPEC), which is funded from the Computational Chemical Sciences Program by the U.S. Department of Energy (DOE), at Pacific Northwest National Laboratory. The computations were performed on resources provided by the Wigner RCP and by the Swedish National Infrastructure for Computing (SNIC 2018/3-625 and SNIC 2019/1-11) at the National Supercomputer Centre (NSC).
* barcza.gergely@wigner.mta.hu

1 Tran, T. T.; Bray, K.; Ford, M. J.; Toth, M.; Aharonovich, I. Quantum emission from hexagonal boron nitride monolayers. Nat. Nanotechnol. 2015, 11, 37.

2 Tran, T. T.; Elbadawi, C.; Totonjian, D.; Lobo, C. J.; Grosso, G.; Moon, H.; Englund, D. R.; Ford, M. J.;
Aharonovich, I.; Toth, M. Robust Multicolor Single Photon Emission from Point Defects in Hexagonal Boron Nitride. ACS Nano 2016, 10, 7331-7338.

3 Chejanovsky, N.; Rezai, M.; Paolucci, F.; Kim, Y.; Rendler, T.; Rouabeh, W.; Fávaro de Oliveira, F.; Herlinger, P.; Denisenko, A.; Yang, S.; Gerhardt, I.; Fin- 
kler, A.; Smet, J. H.; Wrachtrup, J. Structural Attributes and Photodynamics of Visible Spectrum Quantum Emitters in Hexagonal Boron Nitride. Nano Lett. 2016, 16, 7037-7045.

4 Jungwirth, N. R.; Calderon, B.; Ji, Y.; Spencer, M. G.; Flatté, M. E.; Fuchs, G. D. Temperature Dependence of Wavelength Selectable Zero-Phonon Emission from Single Defects in Hexagonal Boron Nitride. Nano Lett. 2016, 16 , 6052-6057.

5 Exarhos, A. L.; Hopper, D. A.; Grote, R. R.; Alkauskas, A.; Bassett, L. C. Optical Signatures of Quantum Emitters in Suspended Hexagonal Boron Nitride. ACS Nano 2017, 11, 3328-3336.

${ }^{6}$ Proscia, N. V.; Shotan, Z.; Jayakumar, H.; Reddy, P.; Cohen, C.; Dollar, M.; Alkauskas, A.; Doherty, M.; Meriles, C. A.; Menon, V. M. Near-deterministic activation of room-temperature quantum emitters in hexagonal boron nitride. Optica 2018, 5, 1128-1134.

7 Bommer, A.; Becher, C. New insights into nonclassical light emission from defects in multi-layer hexagonal boron nitride. Nanophotonics 2019, 0, 0123.

8 Gottscholl, A.; Kianinia, M.; Soltamov, V.; Orlinskii, S.; Mamin, G.; Bradac, C.; Kasper, C.; Krambrock, K.; Sperlich, A.; Toth, M.; Aharonovich, I.; Dyakonov, V. Initialization and read-out of intrinsic spin defects in a van der Waals crystal at room temperature. Nat. Mater. 2020, 19, 540-545.

9 Chejanovsky, N.; Mukherjee, A.; Kim, Y.; Denisenko, A.; Finkler, A.; Taniguchi, T.; Watanabe, K.; Bhaktavatsala Rao, D. D.; Smet, J. H.; Wrachtrup, J. Single spin resonance in a van der Waals embedded paramagnetic defect. 2019; arXiv:1906.05903. arXiv.org e-Print archive. https://arxiv.org/abs/1906.05903 (accessed 13 June 2019).

10 Orellana, W.; Chacham, H. Stability of native defects in hexagonal and cubic boron nitride. Phys. Rev. B 2001, 63, 125205.

11 Piquini, P.; Baierle, R. J.; Schmidt, T. M.; Fazzio, A. Formation energy of native defects in BN nanotubes: anab initiostudy. Nanotechnology 2005, 16, 827-831.

$12 \mathrm{Si}$, M. S.; Xue, D. S. Magnetic properties of vacancies in a graphitic boron nitride sheet by first-principles pseudopotential calculations. Phys. Rev. B 2007, 75, 193409.

13 Attaccalite, C.; Bockstedte, M.; Marini, A.; Rubio, A.; Wirtz, L. Coupling of excitons and defect states in boronnitride nanostructures. Phys. Rev. B 2011, 83, 144115.

${ }^{14}$ Huang, B.; Lee, H. Defect and impurity properties of hexagonal boron nitride: A first-principles calculation. Phys. Rev. B 2012, 86, 245406.

15 Zhang, Z.-F.; Zhou, T.-G.; Zuo, X. First-principles calculations of h-BN monolayers by doping with oxygen and sulfur. Acta Physica Sinica 2013, 62, 83102.

16 Cheng, G.; Zhang, Y.; Yan, L.; Huang, H.; Huang, Q.; Song, Y.; Chen, Y.; Tang, Z. A paramagnetic neutral CBVN center in hexagonal boron nitride monolayer for spin qubit application. Comput. Mater. Sci. 2017, 129, $247-251$.

17 Tawfik, S. A.; Ali, S.; Fronzi, M.; Kianinia, M.; Tran, T. T.; Stampfl, C.; Aharonovich, I.; Toth, M.; Ford, M. J. First-principles investigation of quantum emission from hBN defects. Nanoscale 2017, 9, 1357513582 .

18 Weston, L.; Wickramaratne, D.; Mackoit, M.; Alkauskas, A.; Van de Walle, C. G. Native point defects and impurities in hexagonal boron nitride. Phys. Rev. B 2018, 97, 214104.

19 Abdi, M.; Chou, J.-P.; Gali, A.; Plenio, M. B. Color Centers in Hexagonal Boron Nitride Monolayers: A Group Theory and Ab Initio Analysis. ACS Photonics 2018, 5, 1967-1976.

20 Sajid, A.; Reimers, J. R.; Ford, M. J. Defect states in hexagonal boron nitride: Assignments of observed properties and prediction of properties relevant to quantum computation. Phys. Rev. B 2018, 97, 064101.

21 Reimers, J. R.; Sajid, A.; Kobayashi, R.; Ford, M. J. Understanding and Calibrating Density-Functional-Theory Calculations Describing the Energy and Spectroscopy of Defect Sites in Hexagonal Boron Nitride. J. Chem. Theory Comput. 2018, 14, 1602-1613.

22 Sajid, A.; Reimers, J. R.; Thygesen, K. S.; Ford, M. J. Identification of defects responsible for optically detected magnetic resonance in hexagonal boron nitride. 2019; arXiv:1912.07816. arXiv.org e-Print archive. https://arxiv.org/abs/1912.07816 (accessed 17 Oct 2019).

${ }^{23}$ Kohn, W.; Sham, L. J. Self-Consistent Equations Including Exchange and Correlation Effects. Phys. Rev. 1965, 140, A1133-A1138.

24 Cohen, A. J.; Mori-Snchez, P.; Yang, W. Challenges for Density Functional Theory. Chem. Rev. 2012, 112, 289320 .

${ }^{25}$ Miehlich, B.; Stoll, H.; Savin, A. A correlation-energy density functional for multideterminantal wavefunctions. Mol. Phys. 1997, 91, 527-536.

${ }^{26}$ Grafenstein, J.; Kraka, E.; Cremer, D. Density functional theory for open-shell singlet biradicals. Chem. Phys. Lett. 1998, 288, $593-602$.

27 Grimme, S.; Waletzke, M. A combination of KohnSham density functional theory and multi-reference configuration interaction methods. J. Chem. Phys. 1999, 111, 5645-5655.

${ }^{28} \mathrm{Wu}, \mathrm{W}$; Shaik, S. VB-DFT: a nonempirical hybrid method combining valence bond theory and density functional energies. Chem. Phys. Lett. 1999, 301, $37-42$.

29 Zhao, Y.; Lynch, B. J.; Truhlar, D. G. Multi-coefficient extrapolated density functional theory for thermochemistry and thermochemical kinetics. Phys. Chem. Chem. Phys. 2005, 7, 43-52.

30 Ukai, T.; Nakata, K.; Yamanaka, S.; Takada, T.; Yamaguchi, K. A CAS-DFT study of fundamental degenerate and nearly degenerate systems. Mol. Phys. 2007, 105, 2667-2679.

31 Fromager, E.; Toulouse, J.; Jensen, H. J. A. On the universality of the long-/short-range separation in multiconfigurational density-functional theory. J. Chem. Phys. 2007, 126, 074111.

32 Ying, F.; Su, P.; Chen, Z.; Shaik, S.; Wu, W. DFVB: A Density-Functional-Based Valence Bond Method. J. Chem. Theory Comput. 2012, 8, 1608-1615.

33 Roemelt, M.; Maganas, D.; DeBeer, S.; Neese, F. A combined DFT and restricted open-shell configuration interaction method including spin-orbit coupling: Application to transition metal L-edge X-ray absorption spectroscopy. J. Chem. Phys. 2013, 138, 204101.

34 Li Manni, G.; Carlson, R. K.; Luo, S.; Ma, D.; Olsen, J.; Truhlar, D. G.; Gagliardi, L. Multiconfiguration PairDensity Functional Theory. J. Chem. Theory Comput. 2014, 10, 3669-3680. 
35 Roos, B. O. Adv. Chem. Phys.; John Wiley and Sons, Ltd: New York, 1987; pp 399-445.

36 Shepard, R. Adv. Chem. Phys.; John Wiley and Sons, Ltd: New York, 1987; pp 63-200.

37 Sherrill, C. D.; Schaefer, H. F. In The Configuration Interaction Method: Advances in Highly Correlated Approaches; Lowdin, P.-O., Sabin, J. R., Zerner, M. C., Brandas, E., Eds.; Advances in Quantum Chemistry; Academic Press, 1999; Vol. 34; pp 143 - 269.

38 Werner, H.-J. Adv. Chem. Phys.; John Wiley and Sons, Ltd, 1987; pp 1-62.

39 Shamasundar, K. R.; Knizia, G.; Werner, H.-J. A new internally contracted multi-reference configuration interaction method. J. Chem. Phys. 2011, 135, 054101.

40 Austin, B. M.; Zubarev, D. Y.; Lester, W. A. Quantum Monte Carlo and Related Approaches. Chem. Rev. 2012, 112, 263-288.

41 Szalay, P. G.; Muller, T.; Gidofalvi, G.; Lischka, H.; Shepard, R. Multiconfiguration Self-Consistent Field and Multireference Configuration Interaction Methods and Applications. Chem. Rev. 2012, 112, 108-181.

42 Helgaker, T.; Jorgensen, P.; Olsen, J. Molecular Electronic Structure Theory; John Wiley and Sons, Ltd, 2014; Chapter 11, pp 523-597.

43 Lischka, H.; Nachtigallova, D.; Aquino, A. J. A.; Szalay, P. G.; Plasser, F.; Machado, F. B. C.; Barbatti, M. Multireference Approaches for Excited States of Molecules. Chem. Rev. 2018, 118, 7293-7361.

44 Shepherd, J. J.; Booth, G. H.; Alavi, A. Investigation of the full configuration interaction quantum Monte Carlo method using homogeneous electron gas models. J. Chem. Phys. 2012, 136, 244101.

45 Booth, G. H.; Grüneis, A.; Kresse, G.; Alavi, A. Towards an exact description of electronic wavefunctions in real solids. Nature 2013, 493, 365-370.

46 McClain, J.; Sun, Q.; Chan, G. K.-L.; Berkelbach, T. C. Gaussian-Based Coupled-Cluster Theory for the GroundState and Band Structure of Solids. J. Chem. Theory Comput. 2017, 13, 1209-1218.

47 Zhang, S.; Malone, F. D.; Morales, M. A. Auxiliary-field quantum Monte Carlo calculations of the structural properties of nickel oxide. J. Chem. Phys. 2018, 149, 164102.

48 Zhang, I. Y.; Grneis, A. Coupled Cluster Theory in Materials Science. Front. Mater. 2019, 6, 123.

49 Malone, F. D.; Zhang, S.; Morales, M. A. Accelerating Auxiliary-Field Quantum Monte Carlo Simulations of Solids with Graphical Processing Units. J. Chem. Theory Comput. 2020, 16, 4286-4297.

50 Benali, A.; Gasperich, K.; Jordan, K. D.; Applencourt, T.; Luo, Y.; Bennett, M. C.; Krogel, J. T.; Shulenburger, L.; Kent, P. R. C.; Loos, P.-F.; Scemama, A.; Caffarel, M. Towards a Systematic Improvement of the FixedNode Approximation in Diffusion Monte Carlo for Solids. 2020; arXiv:physics.chem-ph/2007.11673. arXiv.org ePrint archive. https://arxiv.org/abs/2007.11673 (accessed 29 Oct 2020).

51 Baiardi, A.; Reiher, M. The density matrix renormalization group in chemistry and molecular physics: Recent developments and new challenges. J. Chem. Phys. 2020, 152, 040903.

52 Fertitta, E.; Paulus, B.; Barcza, G.; Legeza, O. Investigation of metal-insulator-like transition through the ab initio density matrix renormalization group approach. Phys. Rev. B 2014, 90, 245129.
53 Ronca, E.; Li, Z.; Jimenez-Hoyos, C. A.; Chan, G. K.L. Time-Step Targeting Time-Dependent and Dynamical Density Matrix Renormalization Group Algorithms with ab Initio Hamiltonians. J. Chem. Theory Comput. 2017, 13, 5560-5571.

${ }^{54}$ White, S. R. Density matrix formulation for quantum renormalization groups. Phys. Rev. Lett. 1992, 69, 28632866.

55 White, S. R. Density-matrix algorithms for quantum renormalization groups. Phys. Rev. B 1993, 48, 1034510356.

56 Xiang, T. Density-matrix renormalization-group method in momentum space. Phys. Rev. B 1996, 53, R10445R10448.

57 Nishimoto, S.; Jeckelmann, E.; Gebhard, F.; Noack, R. M. Application of the density matrix renormalization group in momentum space. Phys. Rev. B 2002, 65, 165114.

58 Legeza, Ö.; Sólyom, J. Optimizing the density-matrix renormalization group method using quantum information entropy. Phys. Rev. B 2003, 68, 195116.

59 White, S. R.; Martin, R. L. Ab initio quantum chemistry using the density matrix renormalization group. J. Chem. Phys. 1999, 110, 4127-4130.

60 Chan, G. K.-L.; Head-Gordon, M. Highly correlated calculations with a polynomial cost algorithm: A study of the density matrix renormalization group. J. Chem. Phys. 2002, 116, 4462-4476.

61 Legeza, Ö.; Röder, J.; Hess, B. A. Controlling the accuracy of the density-matrix renormalization-group method: The dynamical block state selection approach. Phys. Rev. B 2003, 67, 125114.

${ }^{62}$ Rissler, J.; Noack, R. M.; White, S. R. Measuring orbital interaction using quantum information theory. Chem. Phys. 2006, 323, 519 - 531.

63 Barcza, G.; Legeza, Ö.; Marti, K. H.; Reiher, M. Quantum-information analysis of electronic states of different molecular structures. Phys. Rev. A 2011, 83, 012508 .

64 Szalay, Sz.; Pfeffer, M.; Murg, V.; Barcza, G.; Verstraete, F.; Schneider, R.; Legeza, Ö. Tensor product methods and entanglement optimization for ab initio quantum chemistry. Int. J. Quantum Chem. 2015, 115, 1342-1391.

65 Schollwöck, U. The density-matrix renormalization group. Rev. Mod. Phys. 2005, 77, 259-315.

66 Schollwöck, U. The density-matrix renormalization group in the age of matrix product states. Ann. Phys. 2011, 326, 96 - 192, January 2011 Special Issue.

67 Olivares-Amaya, R.; Hu, W.; Nakatani, N.; Sharma, S.; Yang, J.; Chan, G. K.-L. The ab-initio density matrix renormalization group in practice. J. Chem. Phys. 2015, 142.

68 Dukelsky, J.; Pittel, S.; Dimitrova, S. S.; Stoitsov, M. V. Density matrix renormalization group method and largescale nuclear shell-model calculations. Phys. Rev. C 2002, 65, 054319.

69 Papenbrock, T.; Dean, D. J. Density matrix renormalization group and wavefunction factorization for nuclei. $J$. Phys. G: Nucl. Part. Phys. 2005, 31, S1377-S1383.

70 Rotureau, J.; Michel, N.; Nazarewicz, W.; Płoszajczak, M.; Dukelsky, J. Density Matrix Renormalization Group Approach for Many-Body Open Quantum Systems. Phys. Rev. Lett. 2006, 97, 110603. 
71 Legeza, Ö.; Veis, L.; Poves, A.; Dukelsky, J. Advanced density matrix renormalization group method for nuclear structure calculations. Phys. Rev. C 2015, 92, 051303.

72 Legeza, O.; Schilling, C. Role of the pair potential for the saturation of generalized Pauli constraints. Phys. Rev. A 2018, 97, 052105.

73 Shapir, I.; Hamo, A.; Pecker, S.; Moca, C. P.; Legeza, O.; Zarand, G.; Ilani, S. Imaging the Wigner Crystal of Electrons in One Dimension. Science 2019, 364, 870.

74 Moca, C. P.; Izumida, W.; Dóra, B.; Legeza, O.; Asbóth, J. K.; Zaránd, G. Topologically Protected Correlated End Spin Formation in Carbon Nanotubes. Phys. Rev. Lett. 2020, 125, 056401.

75 Knecht, S.; Legeza, .; Reiher, M. Communication: Fourcomponent density matrix renormalization group. $J$. Chem. Phys. 2014, 140, 041101.

76 Battaglia, S.; Keller, S.; Knecht, S. Efficient Relativistic Density-Matrix Renormalization Group Implementation in a Matrix-Product Formulation. J. Chem. Theory Comput. 2018, 14, 2353-2369.

77 Brandejs, J.; Visnak, J.; Veis, L.; Mate, M.; Legeza, O.; Pittner, J. Toward DMRG-tailored coupled cluster method in the 4c-relativistic domain. J. Chem. Phys. 2020, 152, 174107.

78 Cramer, C. Essentials of Computational Chemistry: Theories and Models; Wiley, 2005.

79 Jensen, F. Introduction to Computational Chemistry; John Wiley and Sons, Inc.: Hoboken, NJ, USA, 2006.

80 Sharma, S.; Sivalingam, K.; Neese, F.; Chan, G. K.-L. Low-energy spectrum of iron-sulfur clusters directly from many-particle quantum mechanics. Nat. Chem. 2014, 6, 927-933.

81 Kawakami, T.; Sano, S.; Saito, T.; Sharma, S.; Shoji, M.; Yamada, S.; Takano, Y.; Yamanaka, S.; Okumura, M.; Nakajima, T.; Yamaguchi, K. UNO DMRG CASCI calculations of effective exchange integrals for m-phenylenebis-methylene spin clusters. Mol. Phys. 2017, 115, 21542167.

82 Ivády, V.; Barcza, G.; Thiering, G.; Li, S.; Hamdi, H.; Chou, J.-P.; Legeza, Ö.; Gali, A. Ab initio theory of the negatively charged boron vacancy qubit in hexagonal boron nitride. npj Comput. Mater. 2020, 6, 41.

83 Szabo, A.; Ostlund, N. S. Modern Quantum Chemistry: Introduction to Advanced Electronic Structure Theory, 1st ed.; Dover Publications, Inc.: Mineola, 1996.

${ }^{84}$ Giannozzi, P. et al. QUANTUM ESPRESSO: a modular and open-source software project for quantum simulations of materials. J. Phys.: Condens. Matter 2009, 21, 395502.

85 Giannozzi, P. et al. Advanced capabilities for materials modelling with QUANTUM ESPRESSO. J. Phys.: Condens. Matter 2017, 29, 465901.

${ }^{86}$ Kresse, G.; Hafner, J. Norm-conserving and ultrasoft pseudopotentials for first-row and transition elements. $J$. Phys.: Condens. Matter 1994, 6, 8245-8257.

87 Gygi, F.; Baldereschi, A. Self-consistent Hartree-Fock and screened-exchange calculations in solids: Application to silicon. Phys. Rev. B 1986, 34, 4405-4408.

88 Östlund, S.; Rommer, S. Thermodynamic Limit of Density Matrix Renormalization. Phys. Rev. Lett. 1995, 75, 3537-3540.

89 Barcza, G.; Barford, W.; Gebhard, F.; Legeza, O. Excited states in polydiacetylene chains: A density matrix renormalization group study. Phys. Rev. B 2013, 87, 245116.
${ }^{90} \mathrm{Hu}, \mathrm{W}$; Chan, G. K.-L. Excited-State Geometry Optimization with the Density Matrix Renormalization Group, as Applied to Polyenes. J. Chem. Theory Comput. 2015, 11, 3000-3009.

91 Bockstedte, M.; Schütz, F.; Garratt, T.; Ivády, V.; Gali, A. Ab initio description of highly correlated states in defects for realizing quantum bits. npj Quantum Mater. 2018, 3, 31.

92 Stein, C. J.; Reiher, M. Automated Selection of Active Orbital Spaces. J. Chem. Theory Comput. 2016, 12, 17601771.

93 Grüneis, A.; Booth, G. H.; Marsman, M.; Spencer, J.; Alavi, A.; Kresse, G. Natural Orbitals for Wave Function Based Correlated Calculations Using a Plane Wave Basis Set. J. Chem. Theory Comput. 2011, 7, 2780-2785.

94 Møller, C.; Plesset, M. S. Note on an Approximation Treatment for Many-Electron Systems. Phys. Rev. 1934, 46, 618-622.

95 Booth, G. H.; Tsatsoulis, T.; Chan, G. K.-L.; Grneis, A. From plane waves to local Gaussians for the simulation of correlated periodic systems. J. Chem. Phys. 2016, 145, 084111.

96 Zhu, T.; Cui, Z.-H.; Chan, G. K.-L. Efficient Formulation of Ab Initio Quantum Embedding in Periodic Systems: Dynamical Mean-Field Theory. J. Chem. Theory Comput. 2020, 16, 141-153.

97 Sajid, A.; Ford, M. J.; Reimers, J. R. Single-photon emitters in hexagonal boron nitride: a review of progress. Reports on Progress in Physics 2020, 83, 044501.

98 Perdew, J. P.; Burke, K.; Ernzerhof, M. Generalized Gradient Approximation Made Simple. Phys. Rev. Lett. 1996, 77, 3865-3868.

${ }^{99}$ Kresse, G.; Furthmüller, J. Efficient iterative schemes for $a b$ initio total-energy calculations using a plane-wave basis set. Phys. Rev. B 1996, 54, 11169-11186.

100 Neese, F. The ORCA program system. Wiley Interdiscip. Rev.: Comput. Mol. Sci. 2012, 2, 73-78.

101 Kallay, M. et al. Mrcc, a quantum chemical program suite. 2020; www.mrcc.hu.

102 Kallay, M. et al. The MRCC program system: Accurate quantum chemistry from water to proteins. J. Chem. Phys. 2020, 152, 074107.

103 Legeza, Ö.; Veis, L.; Mosoni, T. QC-DMRG-Budapest, a program for quantum chemical DMRG calculations. 2020.

104 Guo, S.; Watson, M. A.; Hu, W.; Sun, Q.; Chan, G. K.L. N-Electron Valence State Perturbation Theory Based on a Density Matrix Renormalization Group Reference Function, with Applications to the Chromium Dimer and a Trimer Model of Poly(p-Phenylenevinylene). J. Chem. Theory Comput. 2016, 12, 1583-1591.

105 Reimers, J. R.; Sajid, A.; Kobayashi, R.; Ford, M. J. Convergence of Defect Energetics Calculations. J. Phys. Chem. C 2020, 124, 21178-21183. 


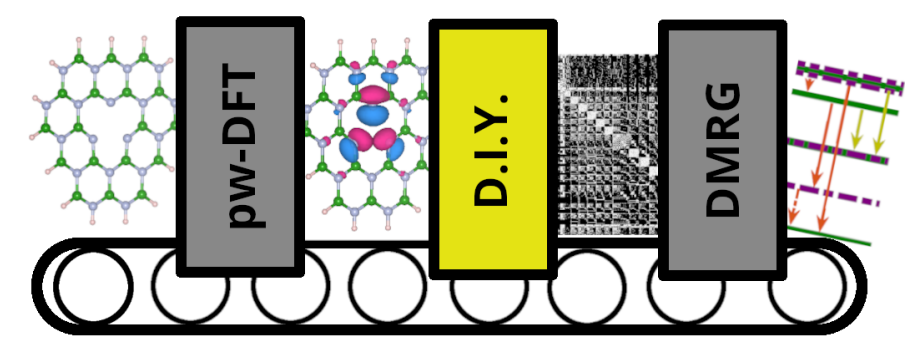

